\title{
Replacement of calcified ascending aorta in patients undergoing aortic valve replacement
}

\author{
Hyoung Woo Chang, Dong Seop Jeong, Yang Hyun Cho, Kiick Sung, Wook-Sung Kim, Young Tak Lee, \\ Pyo Won Park
}

Department of Thoracic and Cardiovascular Surgery, Samsung Medical Center, Sungkyunkwan University School of Medicine, Seoul, Republic of Korea

Contributions: (I) Conception and design: PW Park, HW Chang; (II) Administrative support: PW Park; (III) Provision of study materials or patients: PW Park; (IV) Collection and assembly of data: HW Chang, PW Park; (V) Data analysis and interpretation: HW Chang, PW Park; (VI) Manuscript writing: All authors; (VII) Final approval of manuscript: All authors.

Correspondence to: Pyo Won Park, MD, PhD. Department of Thoracic and Cardiovascular Surgery, Samsung Medical Center, Sungkyunkwan University School of Medicine, 81 Irwon-ro, Gangnam-gu, Seoul 06351, Republic of Korea. Email: pw.park@samsung.com.

Background: Aortic stenosis (AS) with an extensively calcified ascending aorta is a growing indication for transcatheter aortic valve implantation (TAVI) because aortic manipulation during surgical aortic valve replacement (AVR) is unsafe in these patients. The aim of this study was to evaluate the outcomes of AVR plus ascending aorta replacement (AAR) in patients with severe AS and a heavily calcified ascending aorta.

Methods: From 2004 to 2014, a total of 32 patients with severe AS and extensive aortic calcification underwent concomitant first-time AVR and AAR (AVR + AAR). The mean patient age was 74 7 (range, 59-87) years, and 7 (22\%) patients were octogenarians. The mean logistic EuroSCORE was $21.4 \% \pm 19.0 \%$ (range, 3.3-68.2\%). Arterial cannulae were placed at the ascending aorta $(n=26,81 \%)$, aortic arch $(n=5$, $16 \%)$, or axillary artery $(\mathrm{n}=1,3 \%)$. The aorta was not clamped, and circulatory arrest was used in all patients. One-to-many (1:n) propensity score matching between the study population (AVR + AAR, n=29) and control group (isolated AVR for severe AS, n=433) was performed.

Results: There was no early mortality in the study population. Postoperative neurologic complications included a minor stroke, which resolved without sequelae at discharge, and a transient ischemic attack. The 5 -year survival rate was $83 \% \pm 9 \%$. In the propensity score-matched comparison, 5-year survival was not significantly different between groups; $81 \% \pm 10 \%$ in the AVR + AAR group vs. $87 \% \pm 2 \%$ in the isolated AVR group ( $\mathrm{P}=0.950)$.

Conclusions: Surgical AVR with AAR in AS patients with calcified ascending aortas led to acceptable early and late outcomes. Although the applications for TAVI are growing, a surgical approach may be an alternative option for relatively younger patients with severely calcified aorta.

Keywords: Aortic valve stenosis; vascular calcification; thoracic aorta; deep hypothermic circulatory arrest (DHCA)

Submitted May 31, 2017. Accepted for publication Oct 13, 2017.

doi: $10.21037 /$ jtd.2017.10.100

View this article at: http://dx.doi.org/10.21037/jtd.2017.10.100

\section{Introduction}

Surgical aortic valve replacement (AVR) is the standard treatment for severe aortic stenosis (AS). However, with the advent of transcatheter aortic valve implantation (TAVI), treatment of surgically risky patients shifted from AVR to TAVI. Applications for TAVI are increasing as evidence of its effectiveness accumulates. According to the 2014 AHA/ ACC Guidelines for the Management of Patients With Valvular Heart Disease, TAVI is recommended in patients who meet the indications for AVR for AS and have a prohibitive surgical risk (Class I) (1). One of the prohibitive surgical risks is a heavily calcified aorta. According to the report on the reasons for inoperability in the Placement of 
Table 1 Baseline patient characteristics

\begin{tabular}{|c|c|}
\hline Variables & Total $(n=32)$ \\
\hline Age (years) & $74 \pm 7(59-87)$ \\
\hline $50-59$ & $1[3]$ \\
\hline $60-69$ & 8 [25] \\
\hline $70-79$ & $16[50]$ \\
\hline$\geq 80$ & 7 [22] \\
\hline Sex (female) & $11[34]$ \\
\hline Hypertension & $24[75]$ \\
\hline Diabetes mellitus & $17[53]$ \\
\hline Stroke history & $2[6]$ \\
\hline Chronic kidney disease & $5[16]$ \\
\hline Previous myocardial infarction & $2[6]$ \\
\hline Unstable angina & $1[3]$ \\
\hline Stable angina & 8 [25] \\
\hline \multicolumn{2}{|l|}{ NYHA functional class } \\
\hline 1 & $6[19]$ \\
\hline II & $17[53]$ \\
\hline III & 8 [25] \\
\hline IV & $1[3]$ \\
\hline Preoperative inotropic support & $6[19]$ \\
\hline Atrial fibrillation & 7 [22] \\
\hline Bicuspid aortic valve & $5[16]$ \\
\hline Logistic EuroSCORE (\%) & $21.4 \pm 19.0(3.3-68.2)$ \\
\hline
\end{tabular}

Data were presented as number [\%] or mean \pm standard deviation (range). NYHA, New York Heart Association.

Aortic Transcatheter Valve (PARTNER) Trial, a porcelain aorta was the most common (42\%) technical reason (2). This is because a calcified ascending aorta is difficult to manage during surgical AVR; aorta cannulation, aorta cross-clamping, and aortotomy can mobilize atheromatous plaque, allowing debris flow into the arch vessels.

Although the techniques and technologies involved in TAVI have improved, there are still several drawbacks, such as residual aortic regurgitation, high incidence of heart block and uncertain long-term durability of crimped valves (3). On the other hand, recent reports still show high operative mortality in the surgical treatment of patients with AS and severe aortic calcification $(4,5)$. Therefore, choosing between TAVI and surgical AVR among AS patients with heavily calcified aorta is a challenge, especially in Korea, where a patient's economic burden for the TAVI procedure is huge (3 times more expensive) compared to surgical AVR due to inadequate reimbursement from the national insurance.

We employed a surgical strategy of ascending aorta cannulation where possible and limited replacement of the ascending aorta with one branched vascular graft under deep hypothermic circulatory arrest (DHCA) among patients with heavily calcified ascending aorta. The aim of this study was to compare the outcomes of AVR plus ascending aorta replacement (AAR) in patients with both severe AS and a heavily calcified ascending aorta with outcomes in the control group (isolated AVR). Propensity score-matched analyses were performed.

\section{Methods}

From January 2004 to December 2014, a total of 32 patients with severe AS underwent AVR plus AAR under circulatory arrest due to a heavily calcified ascending aorta. Patients who had previously undergone cardiac surgery or exhibited significant aortic regurgitation were excluded from the study.

Medical records were retrospectively reviewed. The institutional review board of the hospital approved this study, and informed consent from patients was waived based on the retrospective nature of the research (IRB No. 2015-04-104). Cohort characteristics are summarized in Table 1 . The mean patient age was $74 \pm 7$ years, and 7 (22\%) patients were octogenarians. The mean logistic EuroSCORE was $21.4 \% \pm 19.0 \%$ (3.3-68.2\%). Seven patients (22\%) had atrial fibrillation. The duration of DHCA was $25 \pm 5$ minutes.

\section{Surgical technique}

Non-contrast cardiac computed tomography (CT) including the aortic arch is our routine screening procedure among all cardiovascular surgical patients over the age of 60 . Since 2008, we have routinely performed non-contrast heart or chest CT in patients with AS older than 50 years of age to detect calcification in the ascending aorta. When significant calcific burden was demonstrated in the ascending aorta (Figures 1,2), we carefully reviewed the distribution of calcification to determine the arterial cannulation site, expected size of branch graft, and graft-to-aorta anastomosis line. Even when an area seemed to be a good candidate for arterial cannulation on the CT scan, we routinely used 

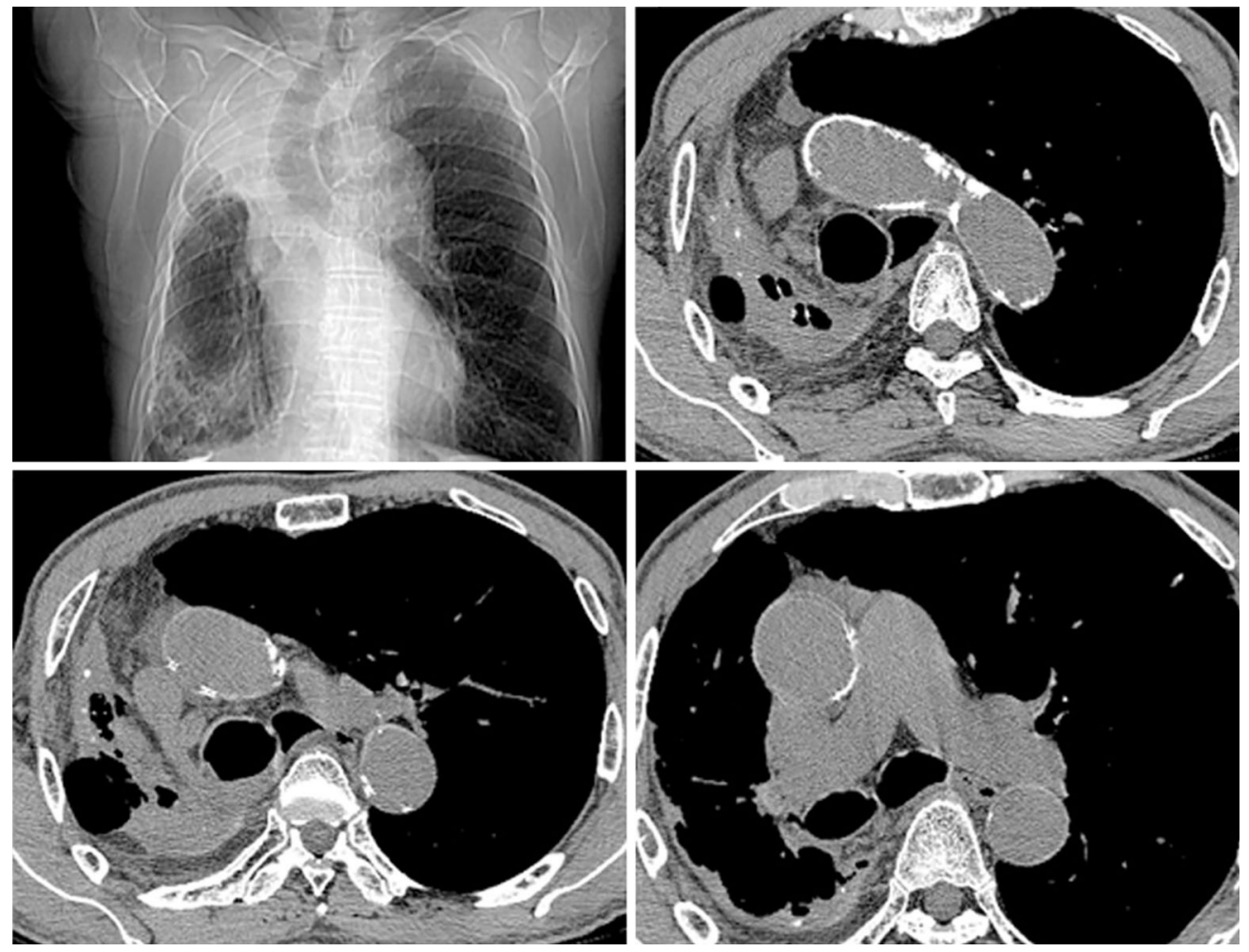

Figure 1 Computed tomography images of an 85-year-old male with severely displaced mediastinum due to tuberculosis. Axillary artery cannulation was performed, and he recovered without incident. He was discharged on postoperative day 8 . He is currently 93 years old and in good health.

intraoperative epiaortic ultrasound to avoid soft noncalcified atheromatous plaques. When cannulation of the ascending aorta was impossible, the lesser curvature of the proximal aortic arch or the right axillary artery was used.

After starting cardiopulmonary bypass $(\mathrm{CPB})$, the patient was cooled down to $20-23{ }^{\circ} \mathrm{C}$ (measured rectally), and a left atrial vent was inserted via the right upper pulmonary vein as heart rate slowed. During the cooling period, we selected a properly sized homemade or commercially available single-branched vascular graft. When the heart beat was almost stopped, right atriotomy was performed. A purse-string suture was made around the coronary sinus for direct retrograde cardioplegia infusion. We used retrograde cold blood cardioplegia in all study patients. Cardioplegia was administered every 25-30 minutes. Anterograde cardioplegia was not used. Immediately before the initiation of circulatory arrest, the patient was placed in the Trendelenburg position. Distal graft-to-aorta anastomosis was performed under circulatory arrest.

We carefully chose an optimal location for distal graftto-aorta anastomosis depending on the distribution of atheromatous plaques. In certain cases, an oblique (beveled) anastomosis line was made to avoid endarterectomy, which might be time consuming during circulatory arrest. However, in some patients with a porcelain aorta, meticulous endarterectomy was necessary.

The chosen distal suture line was very close (within $1 \mathrm{~cm}$ ) to the origin of the $8-\mathrm{mm}$ branch graft to allow space for AVR after clamping. Additional pledget-buttressed sutures were applied to the inside of the posterior anastomotic line to prevent bleeding. After finishing the distal anastomosis, retrograde cerebral perfusion was initiated in the Trendelenburg position with a flow of $1.0-1.2 \mathrm{~L} / \mathrm{min}$ to remove air and calcium debris. Then, a systemic perfusion line was connected to the branch graft.

After restarting anterograde arterial perfusion, we clamped the graft just below the branch graft origin to allow sufficient space for AVR. The ascending aorta was replaced with a Vascutek Gelweave (Terumo Medical Corporation, Somerset, NJ, USA) or Hemashield (Maquet Cardiovascular, Wayne, NJ, USA) graft. After AVR, proximal graft-to-aorta anastomosis was performed. In cases with concomitant coronary artery 

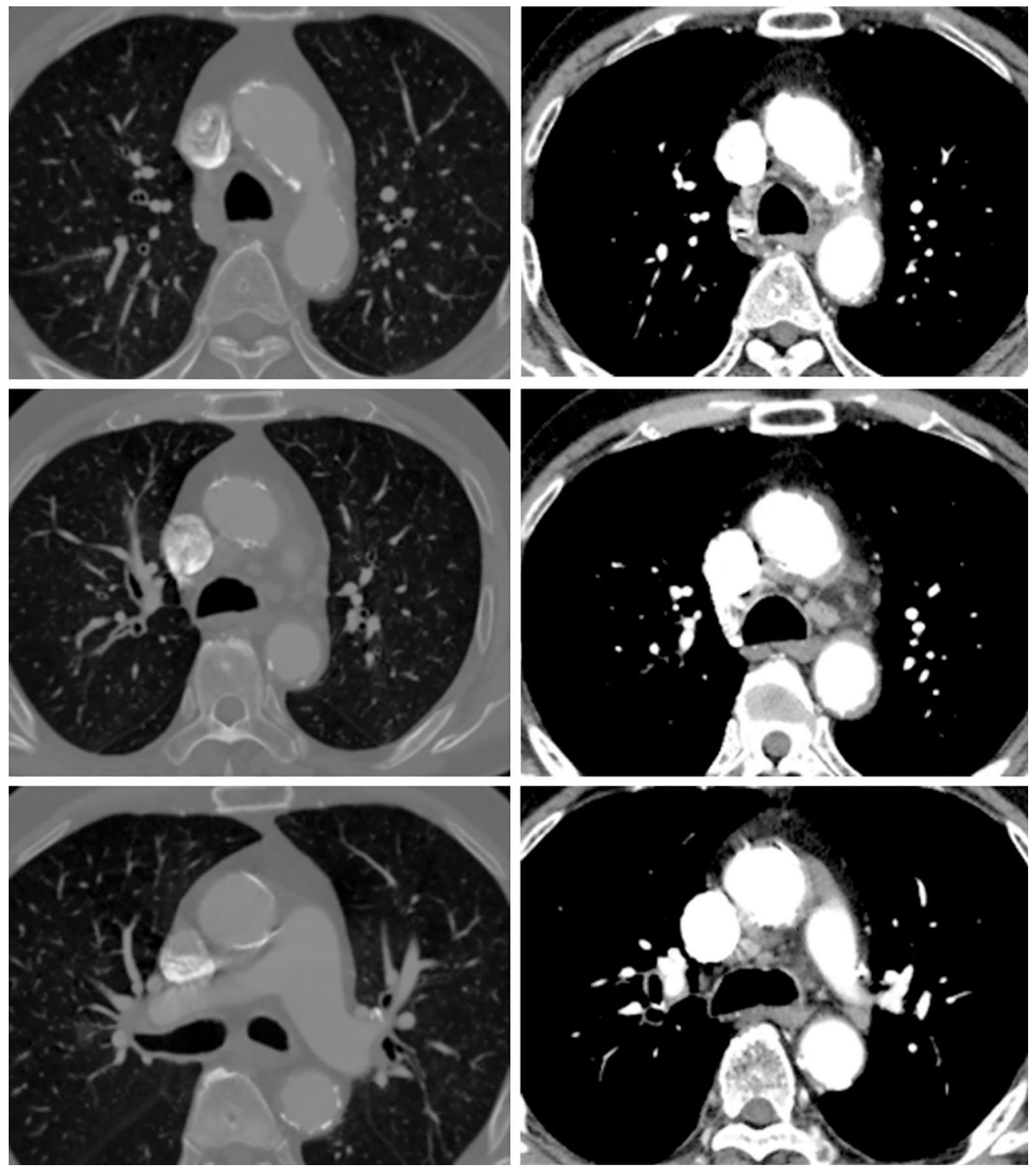

Figure 2 Computed tomography images taken from an 87-year-old male whose calculated EuroSCORE was $68.2 \%$. This patient underwent emergency aortic valve replacement due to progressive orthopnea and acute renal failure. Although he did not show any neurologic complications postoperatively, he died 3 years postoperatively after a prolonged hospital stay.

bypass grafting (CABG), the left internal thoracic artery (ITA) or both ITAs in a Y-structure was used. Conventional and modified ultrafiltration was used in all patients.

\section{Statistical analyses}

Statistical analyses were performed by the Biostatistics support team in the Samsung Biomedical Research Institute. SPSS software, version 20 (IBM, Armonk, NY, USA) and SAS software, version 9.3 (SAS Institute Inc., NC, USA) were used. In addition to basic statistical analyses, we used a valve surgery database to compute comparative survival rates. Of 440 patients who had undergone primary AVR without AAR, 433 patients were matched with 29 out of 32 study patients (AVR + AAR) (Figure 3). There were 23 matching variables (1:n matching), including age, sex, diabetes, hypertension, previous cerebrovascular accident, smoking, chronic obstructive pulmonary disease, chronic kidney disease, coronary artery disease, previous myocardial infarction, New York Heart Association (NYHA) functional 


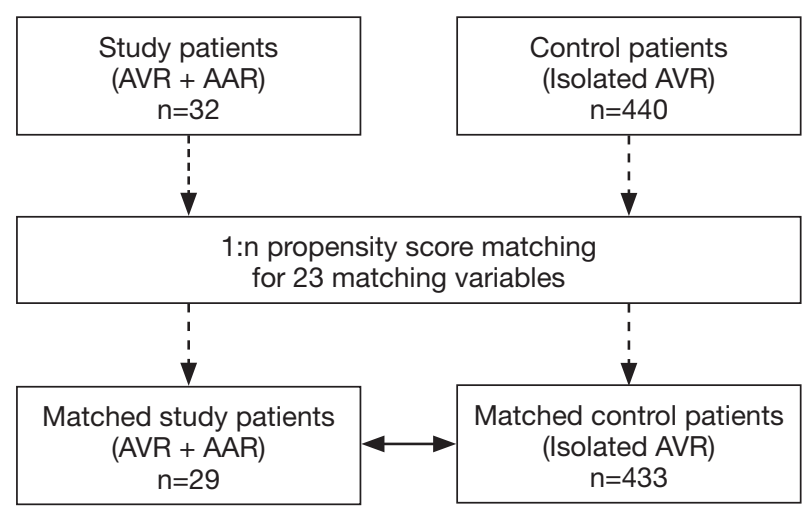

Figure 3 Diagram showing how the propensity score matching was planned. AVR, aortic valve replacement; AAR, ascending aorta replacement.

class, preoperative use of intravenous inotropics, concomitant CABG, Maze operation, atrial fibrillation, body surface area, mitral regurgitation (mild+), tricuspid regurgitation (mild+), aortic valve pressure gradient, left ventricular dysfunction, prosthetic valve size, tricuspid annuloplasty, and concomitant myectomy. After matching, differences in the baseline characteristics were neutralized (Table 2). Overall survival was compared between the matched groups using Cox regression for weighted and clustered data. The weighted Kaplan-Meier method was used to draw survival curves. A $\mathrm{P}$ value $<0.05$ was considered statistically significant.

\section{Results}

Operative data are presented in Table 3. An arterial cannula was placed in the ascending aorta in $26(81 \%)$ patients. The cannula was placed in the aortic arch in $5(16 \%)$ patients and in the right axillary artery in $1(3 \%)$ patient. Hypothermic circulatory arrest was used in all patients with average lowest rectal temperatures of $22.0 \pm 2.0^{\circ} \mathrm{C}$; there was an increase in the lowest rectal temperature between the first 16 patients and the last 16 patients $(21.6 \pm 1.7 \mathrm{vs}$. $\left.22.4 \pm 1.8^{\circ} \mathrm{C}, \mathrm{P}=0.194\right)$. The mean duration of circulatory arrest was $25 \pm 5$ minutes. Circulatory arrest time differed significantly between the first and second half of patients; $27.4 \pm 5.6$ minutes in the first 16 patients vs. $22.2 \pm 3.3$ minutes in the last 16 patients, $\mathrm{P}=0.003$.

\section{Early results}

Early results are summarized in Table 4. The mean intensive care unit (ICU) stay was $6.0 \pm 18.0(0.9-106.0)$ days. Excluding an outlier who stayed in the ICU for 106 days, the mean ICU stay was $2.7 \pm 1.3$ (range, 0.9-5.7) days. The patient who stayed in the ICU for 106 days was an 87-year-old male smoker with diabetes, hypertension, atrial fibrillation, renal dysfunction, coronary artery disease and chronic obstructive pulmonary disease. He had symptoms of left ventricular dysfunction (ejection fraction: $25 \%$ ) and was on inotropic support preoperatively. His calculated logistic EuroSCORE was $68.2 \%$. He was the father of a senior cardiologist in our hospital and had been on the TAVI waiting list twice, but we had to perform emergency AVR due to severe orthopnea and acute renal failure. Although no neurologic complications occurred postoperatively, he needed temporary support with renal replacement therapy. On postoperative day 107 , he moved to a general ward and died 3 years later after a prolonged hospital stay (Figure 2). No patient died within 30 days of the operation. Postoperative neurologic complications included one minor stroke, which resolved without sequela at discharge, and one transient ischemic attack. There were two pacemaker insertions (one for heart block and the other for sick sinus syndrome after a Maze procedure), one exploration for bleeding and one acute renal failure requiring renal replacement therapy. After matching between the AVR + AAR and isolated AVR groups, no significant differences were observed for early adverse outcomes, including early death and stroke (Table 5).

\section{Late outcomes}

The mean follow-up duration in the study population was $2.7 \pm 2.1$ (range, $0-9.4$ ) years. One-year and 5-year survival were $95 \% \pm 5 \%$ and $83 \% \pm 9 \%$, respectively. Overall survival was compared between the matched groups: the study population (AVR + AAR group, $n=29$ ) and a control group that was drawn from our AVR database (isolated AVR group, $n=433$ ). All patients in the matched control group had undergone primary AVR due to severe AS without replacement of the ascending aorta (isolated AVR). Survival curves (Kaplan-Meier analysis) were similar in both groups (Figure 4). Cox regression for weighted and clustered data showed that there were no significant differences between the study population and the matched control group; 5 -year survival was $81 \% \pm 10 \%$ in the AVR + AAR group and $87 \% \pm 2 \%$ in the isolated AVR group $(\mathrm{P}=0.950)$ (Figure 4). 
Table 2 Propensity score-matching data

\begin{tabular}{|c|c|c|c|c|c|c|}
\hline Variables & \multicolumn{3}{|c|}{ Before matching } & \multicolumn{3}{|c|}{ After matching } \\
\hline Age (years), mean $\pm S D$ & $74 \pm 7$ & $72 \pm 6$ & $0.2914^{*}$ & $74 \pm 7$ & $74 \pm 6$ & -0.0221 \\
\hline Previous CVA, n [\%] & $2[6]$ & $36[8]$ & -0.0786 & $2[7]$ & $25[6]$ & 0.0456 \\
\hline \multicolumn{7}{|l|}{ Smoking, $\mathrm{n}[\%]$} \\
\hline Never smoker & $11[34]$ & $258[59]$ & $-0.5028^{*}$ & 10 [34] & $158[36]$ & -0.0394 \\
\hline Chronic kidney disease, n [\%] & $5[16]$ & 97 [22] & $-0.1740^{\star}$ & $5[17]$ & $86[20]$ & -0.0698 \\
\hline Coronary artery disease, n [\%] & 9 [28] & 88 [20] & $0.1779^{*}$ & $8[28]$ & $115[27]$ & 0.0203 \\
\hline Previous MI, n [\%] & $2[6]$ & $12[3]$ & $0.1432^{*}$ & $2[7]$ & $25[6]$ & 0.0445 \\
\hline NYHA class III or IV, n [\%] & $9[28]$ & $114[26]$ & 0.0485 & 8 [28] & $115[27]$ & 0.0233 \\
\hline Preoperative use of inotropics, $n$ [\%] & $6[19]$ & $22[5]$ & $0.3467^{*}$ & $4[14]$ & 60 [14] & 0.0004 \\
\hline Concomitant CABG, n [\%] & $5[16]$ & $70[16]$ & -0.0077 & $4[14]$ & $71[16]$ & -0.0714 \\
\hline Maze, n [\%] & $3[9]$ & $33[8]$ & 0.0633 & $3[10]$ & $34[8]$ & 0.0837 \\
\hline Preoperative LV EF <40\%, n [\%] & $5[16]$ & $38[9]$ & $0.1894^{*}$ & $3[10]$ & $40[9]$ & 0.0310 \\
\hline Valve size $(\mathrm{mm})$, mean $\pm \mathrm{SD}$ & $21 \pm 2$ & $22 \pm 2$ & $-0.4444^{*}$ & $21 \pm 2$ & $22 \pm 2$ & -0.0797 \\
\hline Myectomy, n [\%] & $3[9]$ & 54 [12] & -0.0804 & $3[10]$ & $53[12]$ & -0.0225 \\
\hline Logistic EuroSCORE, mean \pm SD & $21 \pm 19$ & $8 \pm 9$ & $\mathrm{P}=0.001^{*}$ & $19 \pm 16$ & $11 \pm 10$ & $P=0.010^{*}$ \\
\hline
\end{tabular}

*, a standardized mean difference in which the absolute value exceeds 0.1 or a $P$ value less than 0.05 . Logistic EuroSCORE was not used for matching. AVR, aortic valve replacement; AAR, ascending aorta replacement; SMD, standardized mean difference; CVA, cerebrovascular accident; MI, myocardial infarction; NYHA, New York Heart Association; CABG, coronary artery bypass grafting; AF, atrial fibrillation; AV, aortic valve; PG, pressure gradient; LV, left ventricular; EF, ejection fraction.

\section{Discussion}

The present study demonstrated that AS patients with calcified ascending aortas could receive surgical AVR + AAR with acceptable early and late outcomes. The outcomes were validated by comparison with a control group in which patients underwent isolated AVR for AS. To overcome differences in baseline patient characteristics, 1 :n propensity score matching was performed between the study patients $(n=29)$ and the control group $(n=433)$. Mortality and 
Table 3 Operative data

\begin{tabular}{|c|c|}
\hline Variables & Total $(n=32)$ \\
\hline \multicolumn{2}{|l|}{ Arterial cannulation site } \\
\hline Ascending aorta & 26 [81] \\
\hline Right axillary artery & $1[3]$ \\
\hline Aortic arch & $5[16]$ \\
\hline Lowest rectal temperature $\left({ }^{\circ} \mathrm{C}\right)$ & $22.0 \pm 2.0(19.0-24.5)$ \\
\hline CPB time (min) & $192 \pm 35(132-262)$ \\
\hline ACC time (min) & $120 \pm 26(76-176)$ \\
\hline DHCA time (min) & $25 \pm 5(17-43)$ \\
\hline \multicolumn{2}{|l|}{ Aortic valve prosthesis } \\
\hline CE Perimount Magna & 29 [91] \\
\hline Sorin Overline & $1[3]$ \\
\hline St. Jude Regent & $2[6]$ \\
\hline \multicolumn{2}{|l|}{ Combined procedure } \\
\hline CABG & $5[16]$ \\
\hline Maze & 3 [9] \\
\hline Tricuspid annuloplasty & $1[3]$ \\
\hline Subaortic myectomy & 3 [9] \\
\hline Focal endarterectomy performed & 9 [28] \\
\hline
\end{tabular}

Data were presented as number [\%] or mean \pm standard deviation (range). CPB, cardiopulmonary bypass; ACC, aortic cross-clamp; DHCA, deep hypothermic circulatory arrest; CE, Carpentier-Edwards; CABG, coronary artery bypass grafting.

Table 4 Early results

\begin{tabular}{lc}
\hline Variables & Total $(\mathrm{n}=32)$ \\
\hline ICU stay (days) & $6.0 \pm 18.0(0.9-106.0)$ \\
Mechanical ventilation (hours) & $27 \pm 69(4-405)$ \\
Early mortality & $0[0]$ \\
Re-exploration due to bleeding & $1[3]$ \\
Transient ischemic attack & $1[3]$ \\
Minor Stroke without sequelae & $1[3]$ \\
Complete AV block & $1[3]$ \\
Acute kidney injury requiring CRRT & $1[3]$ \\
Mediastinitis & $0[0]$ \\
\hline
\end{tabular}

Data were presented as number [\%] or mean \pm standard deviation (range). ICU, intensive care unit; AV, atrioventricular; CRRT, continuous renal replacement therapy. morbidity were not significantly different between the AVR + AAR and isolated AVR groups.

Although choosing a treatment strategy for patients with severe AS and a heavily calcified ascending aorta is not a new problem, the issues of surgical AVR in unclampable ascending aortas are worth revisiting because TAVI has been substantially developed as a treatment of choice for such patients. The average early mortality and stroke rate is $7-14 \%$ after surgical AVR in these patients according to previous reports $(2,4-9)$. Therefore, opting for TAVI in these patients is a general trend in western countries. However, because the TAVI procedure is about three-times more expensive (about $\$ 27,000$ ) than surgical AVR (about $\$ 9,000$ ) in Korea, we could not always choose between surgery and TAVI according to documented guidelines. The inclusion/exclusion criteria for TAVI generally followed the international guidelines, but in practice, it was difficult to comply with those guidelines. Although we have been performing the TAVI procedure since 2010 in our center, the total number of TAVI procedures was below 50 cases at the end of the study period. Therefore, surgical AVR with AAR was the only option before 2010. After the introduction of TAVI in our hospital, older patients with poor economic status who refused the high-cost TAVI procedure comprised the main patient population that underwent surgical AVR with AAR. Additionally, we still recommend surgical AVR among selected patients younger than 75 years old.

Idrees and colleagues from the Cleveland Clinic have reported on this trend (9). They described 19 patients in whom AVR was aborted due to incidentally found heavy ascending aorta calcification. All patients had been referred to the Cleveland Clinic from other hospitals. In the Cleveland Clinic, surgical AVR was performed in 7 out of 19 patients, and TAVI was performed in the remaining 12 patients.

There are several surgical methods for the management of heavily calcified ascending aortas during AVR (10). The first is to replace the ascending aorta during DHCA. In this approach, the arterial cannula can be placed at the ascending aorta, aortic arch, or axillary artery. When the body temperature reaches $20-21^{\circ} \mathrm{C}$, DHCA is induced. Then, the ascending aorta is resected and replaced with a vascular graft. The second approach is to make a "safe zone" for aortic cross-clamping via endarterectomy or removal of atheroma (called "direct AVR"). In this approach, hypothermic circulatory arrest is used, but the ascending aorta is not replaced (11). The third solution, which may be 
Table 5 Early results after propensity score matching (1:n matching)

\begin{tabular}{|c|c|c|c|}
\hline Variables & AVR + AAR $(n=29), n$ [\%] & Isolated AVR ( $n=433), n$ [\%] & $P$ value \\
\hline Re-exploration due to bleeding & $0[0]$ & $12[3]$ & 0.370 \\
\hline Any stroke & $1[3]$ & 7 [2] & 0.481 \\
\hline Permanent pacemaker insertion & $2[7]$ & $17[4]$ & 0.432 \\
\hline
\end{tabular}

$\mathrm{AV}$, atrioventricular; CRRT, continuous renal replacement therapy.
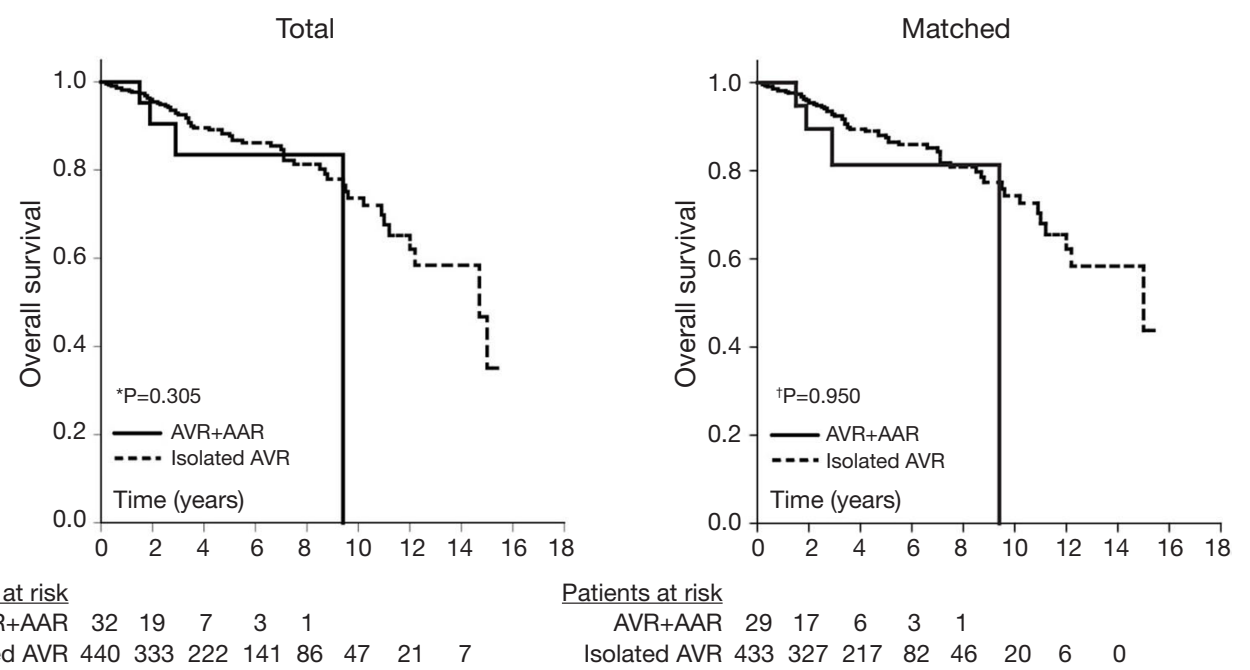

Figure 4 Comparison of overall survival in total and matched patients. * , log-rank test; ${ }^{\dagger}$, cox regression for weighted and clustered data. AVR, aortic valve replacement; AAR, ascending aorta replacement.

considered old-fashioned but is still in use, is bypass from the left ventricular apex to the descending thoracic aorta using a valved conduit.

The results of conventional surgical procedures on the atherosclerotic aorta during surgical AVR are well described in an article by Gillinov and colleagues (6). The sample size in their report was 62 patients. Although their patients were 72 years old on average, hospital mortality was $14 \%$, and stroke occurred in $10 \%$ of the patients. In their paper, patients whose ascending aorta was replaced $(n=12)$ did not experience stroke in the same hospitalization period.

Nishi and colleagues suggested that they could successfully overcome the issue of a heavily calcified ascending aorta by carefully determining the direction of aorta cross-clamping with the help of preoperative CT and epiaortic sonography (12). However, the reproducibility of this approach is questionable as there might not be a "safe direction" of aortic cross-clamping when the calcification covers the whole circumference of the aortic wall.

The study of Kaneko and colleagues included 122 patients (including 44 octogenarians) with unclampable ascending aortas. Their operative mortality was $8 \%$, and stroke incidence was $11 \%$ (5). It is worth noting that they had performed direct AVR under DHCA in some patients in the early period, but they later discarded that strategy because of high stroke incidence

As described above, the surgical methods are heterogeneous, and this makes it difficult to generalize the results of previous reports. The first step in the management of a heavily calcified ascending aorta is detection before surgery. However, a few centers still do not routinely perform chest CT as part of the preoperative work-up. The patients who were referred to the Cleveland Clinic for unexpected porcelain aorta in the report by Idrees et al. did not receive CT scans initially either (9).

In our hospital, we performed non-contrast chest CT 
preoperatively in all patients. With the non-contrast and/or contrast chest CT, we could evaluate the severity and distribution of calcification from the aortic root to the aortic arch. This was very helpful in determining the site for aortic cannulation, cross-clamping, and aortotomy. During the operation, epiaortic ultrasonography was also routinely used to detect noncalcified soft atheroma before cannulation.

The arterial cannula could be placed in the ascending aorta or in the aortic arch in most patients (97\%); however, one patient required right axillary artery cannulation using an 8 -mm vascular graft (Figure 1). This was due to our policy of preferring the ascending aorta or aortic arch for cannulation rather than the axillary artery. In the literature, axillary or femoral arteries are frequently recommended as cannulation sites in patients who need DHCA (13). However, in most cases, it is possible to find a small space in the ascending aorta or aortic arch in which to safely place an arterial cannula. This is consistent with the report of Bapat and colleagues, which showed that there is a proper catheter introduction site on the calcified ascending aorta in more than $99 \%$ of cases during transaortic TAVI (14). Perfusion through the ascending aorta or aortic arch simplifies the whole procedure and can help shorten the surgical duration.

With CPB, the body temperature was lowered to $20-24^{\circ} \mathrm{C}$ and then circulatory arrest was induced. The aorta was not cross-clamped. The ascending aorta was opened and trimmed to allow distal anastomosis. A time-consuming endarterectomy could be minimized by tailoring the distal aortic margin to avoid patch-like dense calcifications. We made every effort to select a clean anterior margin of ascending aorta for the distal anastomosis, because endarterectomy of anterior wall calcification is more difficult, and atheroma debris are invisible after anastomosis. Three or four pledget-buttressed reinforcement sutures were also applied to the posterior side of the distal anastomosis before restarting CPB. Antegrade cerebral perfusion was not used because we did not want to manipulate the atherosclerotic aortic arch vessels. With the accumulation of experience, we reduced the total circulatory arrest time (as presented in the results). Although most of the surgeries were performed by one surgeon (PW Park), other surgeons followed the same strategy in these patients.

Although the average logistic EuroSCORE was 21.4\% and there were 7 (22\%) octogenarians, early mortality was not observed in our patient cohort. There were only one transient ischemic attack and one stroke without permanent sequalae. Excluding an outlier whose logistic EuroSCORE was $68.2 \%$, the average ICU stay was 2.7 days. If this outlier with the extremely high logistic EuroSCORE had been managed with the current state-of-the-art TAVI procedure, the outcomes could have been better than those of surgical AVR. With all these refinements in surgical procedures, the emergence of sutureless AVR may expand the options for patients. A sutureless aortic valve can be implanted during brief DHCA without aortic cross-clamping (15).

There are limitations to our study. First, the sample size is relatively small. The patients in this study had been operated on over a 10-year time span. With strict inclusion criteria, we found 32 out of approximately 500 patients who initially needed isolated AVR for severe AS. Regarding the relatively high incidence of AVR + AAR in our series, the Samsung Medical Center may be accountable, as it is one of the biggest tertiary referral centers in our country. Second, the follow-up duration was not sufficiently long. Third, the patients in our study had relatively low surgical risks aside from a heavily calcified ascending aorta. Although the mean patient age was 74 years and there were $8(22 \%)$ octogenarians, we had a small number of concomitant CABG. In other words, our patients were relatively healthy given their age. Patients with a previous history of cardiac operations in whom the TAVI procedure would be advantageous were also excluded due to their small population size. Thus, further study with a greater number of patients and with homogenous surgical strategies is necessary.

In conclusion, TAVI plays an important role in the treatment of old AS patients with a previous history of cardiac surgery or multiple comorbidities, including severe ascending aorta calcification, because surgical AVR is considered to be too risky for these patients. However, relatively younger patients with severe AS and prohibitive ascending aorta calcification could undergo surgical AVR with acceptable early and late outcomes.

\section{Acknowledgments}

Joo Min Hwang (RN) helped us collect and manage the data. Biomedical statistician Joong Hyun Ahn conducted the statistical analyses.

\section{Footnote}

Conflicts of Interest: Part of this research was presented at the 29th Annual Meeting of the European Association of Cardiothoracic Surgeons, Amsterdam, Netherlands, October 3-7, 2015. 
Ethical Statement: The institutional review board of the hospital approved this study (IRB No. 2015-04-104), and informed consent from patients was waived based on the retrospective nature of the research.

\section{References}

1. Nishimura RA, Otto CM, Bonow RO, et al. 2014 AHA/ ACC Guideline for the Management of Patients With Valvular Heart Disease: A Report of the American College of Cardiology/American Heart Association Task Force on Practice Guidelines. J Am Coll Cardiol 2014;63:e57-185.

2. Makkar RR, Jilaihawi H, Mack M, et al. Stratification of outcomes after transcatheter aortic valve replacement according to surgical inoperability for technical versus clinical reasons. J Am Coll Cardiol 2014;63:901-11.

3. Smith CR, Leon MB, Mack MJ, et al. Transcatheter versus Surgical Aortic-Valve Replacement in High-Risk Patients. N Engl J Med 2011;364:2187-98.

4. Baba H, Goto Y, Ogawa S, et al. The calcified ascending aorta in aortic valve replacement: surgical strategies and results. Gen Thorac Cardiovasc Surg 2014;62:541-6.

5. Kaneko T, Neely RC, Shekar P, et al. The safety of deep hypothermic circulatory arrest in aortic valve replacement with unclampable aorta in non-octogenarians. Interact Cardiovasc Thorac Surg 2015;20:79-84.

6. Gillinov AM, Lytle BW, Hoang V, et al. The atherosclerotic aorta at aortic valve replacement: surgical strategies and results. J Thorac Cardiovasc Surg 2000;120:957-63.

7. Aranki SF, Nathan M, Shekar P, et al. Hypothermic

Cite this article as: Chang HW, Jeong DS, Cho YH, Sung K, Kim WS, Lee YT, Park PW. Replacement of calcified ascending aorta in patients undergoing aortic valve replacement. J Thorac Dis 2017;9(11):4424-4433. doi: 10.21037/jtd.2017.10.100 circulatory arrest enables aortic valve replacement in patients with unclampable aorta. Ann Thorac Surg 2005;80:1679-86; discussion 1686-7.

8. Girardi LN, Krieger KH, Mack CA, et al. No-clamp technique for valve repair or replacement in patients with a porcelain aorta. Ann Thorac Surg 2005;80:1688-92.

9. Idrees J, Roselli EE, Raza S, et al. Aborted sternotomy due to unexpected porcelain aorta: does transcatheter aortic valve replacement offer an alternative choice? J Thorac Cardiovasc Surg 2015;149:131-4.

10. Tajima K. Bad aorta. Gen Thorac Cardiovasc Surg 2014;62:273-81.

11. Takami Y, Tajima K, Terazawa S, et al. Safer aortic crossclamping during short-term moderate hypothermic circulatory arrest for cardiac surgery in patients with a bad ascending aorta. J Thorac Cardiovasc Surg 2009;137:875-80.

12. Nishi H, Mitsuno M, Ryomoto M, et al. Comprehensive approach for clamping severely calcified ascending aorta using computed tomography. Interact Cardiovasc Thorac Surg 2010;10:18-20.

13. Svensson LG, Blackstone EH, Rajeswaran J, et al. Does the arterial cannulation site for circulatory arrest influence stroke risk? Ann Thorac Surg 2004;78:1274-84.

14. Bapat VN, Attia RQ, Thomas M. Distribution of calcium in the ascending aorta in patients undergoing transcatheter aortic valve implantation and its relevance to the transaortic approach. JACC Cardiovasc Interv 2012;5:470-6.

15. Gatti G, Benussi B, Camerini F, et al. Aortic valve replacement within an unexpected porcelain aorta: the sutureless valve option. Interact Cardiovasc Thorac Surg 2014;18:396-8. 